\title{
Hepatotoxicidad inducida por azatioprina en paciente con enfermedad de Crohn
}

\author{
Azathioprine-induced hepatotoxicity in a patient \\ with Crohn's disease \\ D. Ruiz-Clavijo, A. Arín, J.J. Vila, E. Albéniz, R. Iglesias, M. Casi
}

\section{RESUMEN}

Los inmunosupresores se encuentran entre los grupos farmacológicos con mayor potencial teórico de inducir reacciones adversas, entre ellas las hepáticas. Presentamos el caso de un paciente de 35 años con enfermedad de Crohn en tratamiento con azatioprina por corticodependencia al que se le diagnosticó tras realización de una biopsia hepática de hiperplasia nodular regenerativa secundaria a este tratamiento.

Palabras clave. Enfermedad de Crohn. Azatioprina. Hepatotoxicidad. Hiperplasia nodular regenerativa. Hipertensión portal.

\begin{abstract}
Immunosuppressive drugs are among the pharmacological groups with the most theoretical potential to induce adverse reactions, including hepatic reactions. We report the case of a 35-year-old patient with Crohn's disease treated with azathioprine for steroid dependence which, after a hepatic biopsy, was diagnosed with nodular regenerative hyperplasia secondary to this treatment.
\end{abstract}

Key words. Crohn's Disease. Azathioprine. Hepatotoxicity. Nodular regenerative hiperplasia. Portal hipertension.
Servicio de Aparato Digestivo. Complejo Hospitalario de Navarra.

Recepción: 26 de noviembre de 2012

Aceptación provisional: 14 de enero de 2013

Aceptación definitiva: 25 de enero de 2013

\section{Correspondencia:}

David Ruiz-Clavijo

Sección de Aparato Digestivo B

Complejo Hospitalario de Navarra

Irunlarrea, 4

31008 Pamplona

E-mail: davidruizcla@gmail.com 


\section{INTRODUCCIÓN}

El tratamiento con azatioprina, fármaco inmunosupresor de la familia de las tiopurinas, es de uso común en pacientes con enfermedad inflamatoria intestinal. Sus indicaciones fundamentales son: corticodependencia, corticorresistencia, mantenimiento de la remisión, enfermedad fistulizante, y la prevención de la recurrencia post-cirugía ${ }^{1}$. Los efectos secundarios de este fármaco son frecuentes y pueden ser causa de suspensión del tratamiento hasta en un $20 \%$ de pacientes. Actualmente se clasifican en alérgicos o idiosincrásicos y en dosis-dependientes ${ }^{2}$. La hepatotoxicidad se observa hasta en un 3,5\% de los pacientes tratados con tiopurinas y habitualmente se clasifica en tres síndromes fundamentales: síndrome de hipersensibilidad, reacción colestásica idiosincrásica y la hepatotoxicidad dosis-dependiente secundaria a lesión endotelial, que englobaría la hiperplasia nodular regenerativa, la peliosis hepática y la enfermedad venooclusiva $^{3}$.

\section{CASO CLÍNICO}

Paciente de 33 años de edad sin antecedentes de interés, diagnosticado de enfermedad de Crohn ileal a principios de 2005. Siguió tratamiento con claversal $500 \mathrm{mg} / 8 \mathrm{~h}$ y azatioprina a dosis de $150 \mathrm{mg} /$ día desde diciembre de 2005 por corticodependencia. Asimismo estaba en tratamiento con hierro oral en tandas trimestrales, vitamina B12 intramuscular mensual y ácido fólico dos días a la semana. Desde entonces el paciente se encontraba asintomático. En enero de 2007 se objetivó un aumento de las enzimas de colestasis mantenido en el tiempo, decidiendo ampliar el estudio de forma ambulatoria y retirar la azatioprina.

Exploración física: Tensión arterial: 100/60 mm/seg; Pulso: $70 \mathrm{lpm}$; Temperatura: $36^{\circ} \mathrm{C}$; Peso: $80 \mathrm{~kg}$; Talla: $180 \mathrm{~cm}$; Buen estado general. Consciente y orientado. Auscultación cardiopulmonar normal. Abdomen blando, depresible, no doloroso, no masas, ni megalias. Extremidades inferiores sin alteraciones.

Ante la persistencia de la colestasis se realizan:

Analítica. Hemograma y coagulación normal. Plaquetas: 108.000/ml. Perfil hepático: Transaminasas normales. FA: 248U/L. GGT: 390U/L. Perfil férrico y autoinmunidad negativos. Serologías virus de hepatitis $\mathrm{B}$ y $\mathrm{C}$ negativas.

Ecografía abdominal. Hígado aumentado difusamente de tamaño, con contorno liso y parénquima difusamente tosco, heterogéneo e hiperecogénico, sin lesiones ocupacionales. Leve esplenomegalia. Calibre y permeabilidad de la porta normal. Resto de estructuras sin alteraciones.

RMN abdominal. Hígado aumentado difusamente de tamaño, contorno capsular liso y parénquima homogéneo en las secuencias T1 de nivel medio, en las secuencias T2 aparece hipointenso, con áreas parcheadas difusas, discretamente hiperintensas, sin lesiones ocupacionales. Leve esplenomegalia. Resto de estructuras sin alteraciones.

Gastroscopia. En tercio inferior de esófago se observan 3 cordones varicosos pequeños que desaparecen con la insuflación.

Ante la persistencia de colestasis disociada a pesar de la retirada de la azatioprina se decidió realizar una biopsia hepática en la que se aprecia una arquitectura general conservada. Algunos espacios porta están ensanchados con puenteo incompleto porto-portal. Venas centrolobulillares aisladas. No se aprecia colestasis ni hemosiderosis. No macrófagos. PAS diastasa positivo. Algunos espacios porta presentan un ligero infiltrado de células redondas que no desborda la placa limitante. Las trabéculas de hepatocitos irregulares presentan un patrón en zonas regenerativo y en zonas pavimentoso. Aparecen frecuentes células binucleadas, estructuras vasculares y zonas sinusoidales dilatadas con patrón peliótico. Se aprecia un armazón de reticulina irregular con áreas de colapso. La histología descrita anteriormente es compatible con hiperplasia nodular regenerativa. (Figs. 1,2).

La evolución posterior, tanto analítica como de datos de hipertensión portal, fue buena con normalidad analítica y desaparición de las varices esofágicas.

\section{DISCUSIÓN}

La azatioprina y sus análogos (6-mercaptopurina y 6-tioguanina), son inmunomoduladores tiopurínicos con potente efecto inmunosupresor y citostático, empleados en gran cantidad de enfermedades de base autoinmune entre ellas la enfermedad inflamatoria intestinal. Existen dos situaciones muy relevantes en el manejo clínico de los pacientes con enfermedad inflamatoria intestinal donde están indicados: la corticodependencia y la corticorresistencia ${ }^{1}$. El 


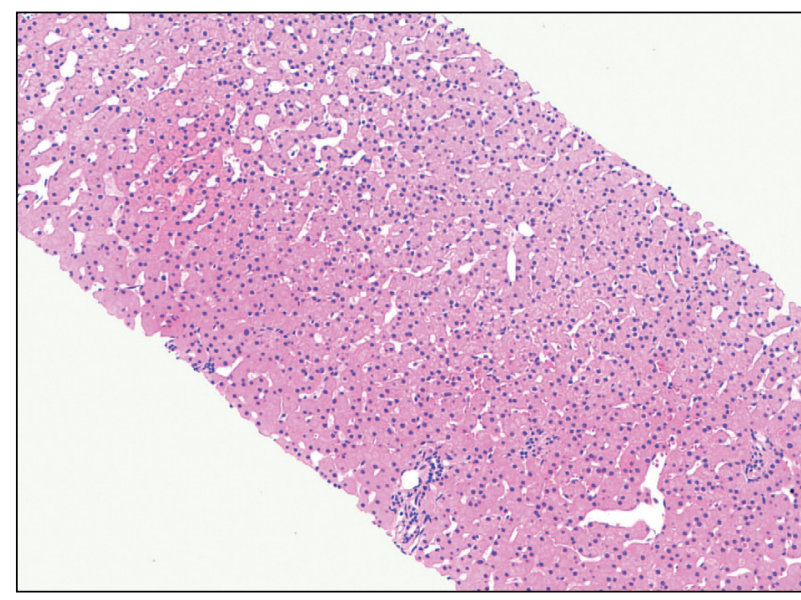

Figura 1. H-E: Áreas de aspecto colapsadas y áreas con importante dilatación de los sinusoides.

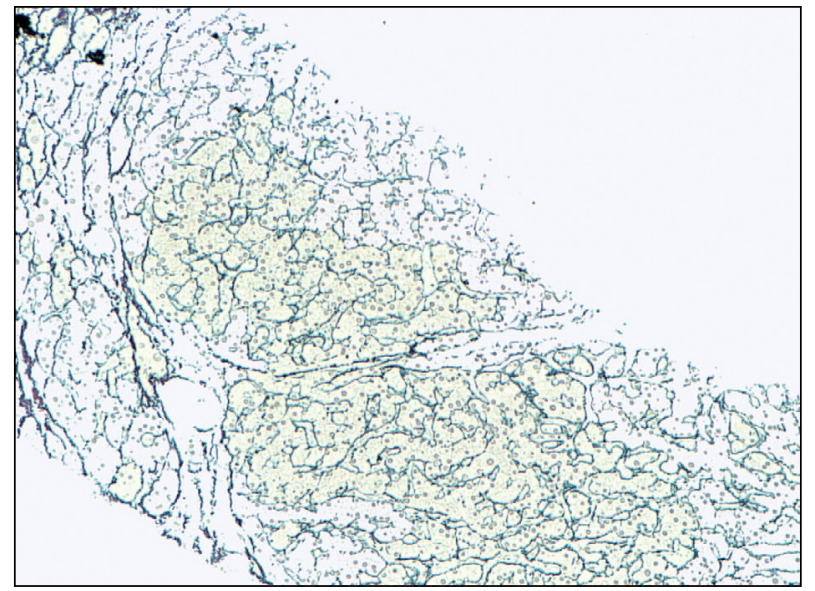

Figura 2. Reticulina: Áreas de puenteo porto-portal incompletas con zonas de trabéculas hepatocitarias colapsadas. inicio de la respuesta a estos dos fármacos se demora entre dos y tres meses.

Su eficacia clínica está limitada por la aparición de efectos adversos que aparecen entre el $15-30 \%$ de los pacientes y provocan la suspensión del tratamiento en aproximadamente el $10 \%$ de ellos. Los efectos secundarios de las tiopurinas se clasifican en idiosincrásicos y en dosisdependientes; dentro de los primeros, se encuentran las reacciones de hipersensibilidad en forma de fiebre, artralgias y rash -que remedan a un síndrome pseudogripal-, la pancreatitis aguda y algunos casos de toxicidad hepática. Dentro de los segundos destacan la mielosupresión y algunos casos de hepatotoxicidad ${ }^{2}$.
La lesión hepática por tiopurinas ocurre con mayor frecuencia en los primeros meses de tratamiento y puede ser asintomática o manifestarse con síntomas inespecíficos como náuseas, cefalea, astenia o dolor abdominal, que generalmente desaparecen al reducir la dosis o suspender el fármaco ${ }^{3}$.

Durante el tratamiento con azatioprina/6-mercaptopurina se han descrito hepatitis agudas tanto de predominio hepatocelular como colestásico, con una prevalencia del $3 \%$ y una incidencia anual del $1,5 \%{ }^{4}$.

Generalmente, las elevaciones leves de aminotransferasas se normalizan espontáneamente durante el seguimiento, incluso 
manteniendo la misma dosis de tiopurina. Cuando la alteración del perfil hepático es moderada se puede intentar reducir la dosis del fármaco al $50 \%$, y si no se consigue la mejoría se debe suspender el tratamiento, siendo infrecuente que no se resuelva el cuadro tras la retirada del fármaco. Existen varias formas de hepatotoxicidad por tiopurinas, que se clasifican en tres síndromes fundamentales: síndrome de hipersensibilidad, en el que los signos y síntomas de hepatotoxicidad ocurren en las primeras 2-4 semanas de tratamiento; reacción colestásica idiosincrásica, en la que aumentan de forma llamativa la bilirrubina y la fosfatasa alcalina junto a una moderada elevación de aminotransferasas (histológicamente la colestasis se acompaña de un grado variable de necrosis hepatocelular $)^{5}$. El tercer síndrome es el de hepatotoxicidad dosis-dependiente secundaria a lesión endotelial, que engloba la hiperplasia nodular regenerativa, la enfermedad veno-oclusiva y la peliosis hepática ${ }^{6}$.

La hiperplasia nodular regenerativa es un tipo de lesión hepática dosis dependiente caracterizada por el daño de las células endoteliales de los sinusoides y las vénulas hepáticas, que deriva en la oclusión no trombótica de los vasos y la posterior aparición de fibrosis e hipertensión portal $^{7}$. Suele aparecer entre los 3 meses y los 3 años de tratamiento con tiopurinas y la patogénesis exacta es desconocida. La hiperplasia nodular regenerativa puede producir hipertensión portal no cirrótica con aparición de varices esófago-gástricas, esplenomegalia e incluso ascitis. Esto puede ocurrir incluso sin observarse alteraciones analíticas en el perfil hepático. Por lo tanto se debe considerar la realización de biopsia hepática en todos los pacientes con signos de hipertensión portal de origen incierto que estén tomando tiopurinas, aunque no presenten alteraciones analíticas ${ }^{8-9}$. En nuestro caso se realizó la biopsia hepática tanto por los datos de hipertensión portal como por las alteraciones analíticas.

La evolución de la hiperplasia nodular regenerativa al suspender los fármacos tiopurínicos suele ser buena, con normaliza- ción analítica y regresión de los signos de hipertensión portal, aunque la evidencia sobre la historia natural es escasa ${ }^{10}$.

\section{BIBLIOGRAFÍA}

1. Lichtenstein GR, Abreu MT, Cohen R, Tremaine W. American Gastroenterological Association Institute technical review on corticosteroids, inmunomodulators, and infliximab in inflammatory bowel disease. Gastroenterology 2006; 130: 940-987.

2. Toscano E, Cotta J, Robles M, Lucena I, Andrade R. Hepatotoxicity induced by new immunosuppressants. Gastroenterol Hepatol 2010; 33: 54-65.

3. Gisbert JP, González-Lama Y, Maté J. Thiopurine-induced liver injury in patients with inflamatory bowel disease: a sistematyc review. Am J Gastroenterol 2007; 102: 1518-1527.

4. Shaye OA, Yadegari M, Abreu MT, Poordad F, SIMON K, MARTIN, et al. Hepatotoxicity of 6-mercaptopurine (6-MP) and azathioprine (AZA) in adult IBD patients. Am J Gastroenterol 2007; 102: 2488-2494.

5. Tapner MJ, Jones BE, Wu WM, Farrell GC. Toxicity of low dose azathioprine and 6-mercaptupurine in rat hepatocytes. Roles of xanthine oxidase and mitochondrial injury. J Hepatol 2004; 40: 454-463.

6. ARnotT ID, GHosh S. Portal hypertension in the presence of minimal liver damage in Crohn's disease on long-term azathioprine: possible endothelial cell injury. Eur J Gastroenterol Hepatol 2000; 12: 569-573.

7. Seksik P, Mary Jy, Beaugerie L, Lémann M, CoLOMBel JF, Vernier-Massoullle G, Cosnes J. Incidence of nodular regenerative hyperplasia in inflammatory bowel disease patients treated with azathioprine. Inflamm Bowel Dis 2011; 17: 565-572.

8. NAER AH, VAN HAELST U, YAP SH. Nodular regenerative hyperplasia of the liver: an important cause of portal hypertension in noncirrhotic patients. J Hepatol 1991; 12: 94-99.

9. Vernier-Massouille G, Cosnes J, Lemann M, Marteau P, Reinisch W, Laharie D et al. Nodular regenerative hyperplasia in patients with inflammatory bowel disease treated with azathioprine. Gut 2007; 56: 1404-1409.

10. Seiderer J, Zech CJ, Diebold J, Schoenberg SO, BRAnd S, Tillack C et al. Nodular regenerative hyperplasia: a reversible entity associated with azathioprine therapy. Eur J Gastroenterol Hepatol 2006; 18: 553-555. 